Published in final edited form as:

J Comput Graph Stat. 2017 ; 26(1): 1-13. doi:10.1080/10618600.2016.1154063.

\title{
Regression Models For Multivariate Count Data
}

\author{
Yiwen Zhang, \\ Department of Statistics, North Carolina State University, Raleigh, NC 27695-8203 \\ Hua Zhou, \\ Department of Biostatistics, University of California, Los Angeles, Los Angeles, CA 90095-1772 \\ Jin Zhou, and \\ Division of Epidemiology and Biostatistics, University of Arizona, Tucson, AZ 85721-0066 \\ Wei Sun \\ Program in Biostatistics and Biomathematics, Fred Hutchinson Cancer Research Center, Seattle, \\ WA 98109
}

\begin{abstract}
Data with multivariate count responses frequently occur in modern applications. The commonly used multinomial-logit model is limiting due to its restrictive mean-variance structure. For instance, analyzing count data from the recent RNA-seq technology by the multinomial-logit model leads to serious errors in hypothesis testing. The ubiquity of over-dispersion and complicated correlation structures among multivariate counts calls for more flexible regression models. In this article, we study some generalized linear models that incorporate various correlation structures among the counts. Current literature lacks a treatment of these models, partly due to the fact that they do not belong to the natural exponential family. We study the estimation, testing, and variable selection for these models in a unifying framework. The regression models are compared on both synthetic and real RNA-seq data.
\end{abstract}

\section{Keywords}

analysis of deviance; categorical data analysis; Dirichlet-multinomial; generalized Dirichletmultinomial; iteratively reweighted Poisson regression (IRPR); negative multinomial; reduced rank GLM; regularization

\section{Introduction}

Multivariate count data abound in modern application areas such as genomics, sports, imaging analysis, and text mining. When the responses are continuous, it is natural to adopt the multivariate normal model. For multivariate count responses, a common choice is the multinomial-logit model (McCullagh and Nelder, 1983). However, the multinomial model is limiting due to its specific mean-variance structure and the implicit assumption that individual counts in the response vector are negatively correlated. In this article, we examine regression models for multivariate counts with more flexible mean-covariance and correlation structure. Parameter estimation in these models is typically hard because they do not belong to the exponential family. We propose a unifying iteratively reweighted Poisson 
regression (IRPR) method for the maximum likelihood estimation. IRPR is stable, scalable to high dimensional data, and simple to implement using existing software. Testing and regularization methods for these models are also studied. Our methods are implemented in the R package and Matlab toolbox mglm (Zhang and Zhou, 2015).

The remaining of the paper is organized as follows. Section 2 motivates our study with analysis of count data in modern genomics. Section 3 introduces a class of GLM models for multivariate count responses. A unifying maximum likelihood estimation procedure is proposed in Section 4. Testing and regularized estimation are treated in Sections 5 and 6 respectively, followed by numerical examples in Section 7 .

\section{Motivation}

Our study is motivated by the analysis of high-throughput data in genomics. Next generation sequencing technology has become the primary choice for massive quantification of genomic features. The data obtained from sequencing technologies are often summarized by the counts of DNA or RNA fragments within a genomic interval. A prime example is the RNA-seq data.

In most mammalian genomes, one gene is composed of multiple exons and different combinations of exons lead to different protein products. One such exon combination is called an RNA isoform. The left panel of Figure 1 depicts a gene with 3 exons and all possible isoforms for that gene. Current RNA-seq platforms are able to deliver the expression counts of each exon set (Wang et al., 2009). An exon set includes contiguous portions isoforms. Here we use the number of RNA-seq reads from exon sets instead of exons because some RNA-seq fragments may overlap with multiple exons (Sun et al., 2015).

Data for one gene with $d$ exon sets takes the form

\begin{tabular}{ccccccccc}
\hline Subject & Exon Set 1 & Exon Set 2 & $\cdots$ & Exon Set $\boldsymbol{d}$ & Treatment & Gender & Age & $\cdots$ \\
\hline 1 & 15 & 0 & $\cdots$ & 3 & Yes & M & 43 & $\cdots$ \\
2 & 0 & 52 & $\cdots$ & 0 & Yes & F & 35 & $\cdots$ \\
$\vdots$ & & & & & $\vdots$ & & & \\
$n$ & 124 & 45 & $\cdots$ & 73 & No & F & 25 & $\cdots$ \\
\hline
\end{tabular}

The primary interest is to identify differential expression profiles and their relation to the covariates. The total expression of a gene in an individual can be obtained by summing the counts across exon sets. Negative binomial regression models have been developed to associate the total expression count with the covariates of interest (Anders and Huber, 2010; Robinson et al., 2010). This approach ignores the RNA isoform expression. More sophisticated methods have been developed to estimate RNA isoform expression and then assess association between isoform expression and covariates of interest. Nevertheless accounting for RNA isoform estimation uncertainty in the association step is a nontrivial task, since the responses in the association step are obtained from variable selection (Sun et al., 2015). An attractive alternative is to treat exon counts as multivariate responses and fit a 
generalized linear model (GLM). The multinomial-logit model is a popular choice, due to its wide availability in statistical software. However, it assumes negative correlation between counts. Two exon sets may belong to one or a few RNA isoforms, leading to complicated correlation structures among their counts. The middle panel of Figure 1 displays the pairwise scatter plots of exon counts for a pseudogene SPCS2P4 with 5 exon sets and the right panel depicts the corresponding correlations in a grayscale image. Notably the correlations can be both positive and negative.

We simulated RNA-seq read counts based on the mouse gene Phlda3 and the RNA-seq data collected from a mouse study (Sun et al., 2015). This gene has 4 exons and 6 exon sets that have non-zero observed read counts. The number of RNA-seq fragments per exon set was simulated by a negative binomial distribution with the mean equal to a linear combination of underlying RNA isoform expression and with the over-dispersion estimated from RNA-seq data. In the generative model, the RNA isoform expression is associated with covariate treatment, but not with age and gender. $n=200$ observations were generated. We fit a negative binomial regression (NegBin) using the total read counts from the 6 exon sets as responses; we also fit the multinomial-logit (MN) model using the multivariate count vectors as responses. The predictor $\log$ (TotalReads) is included as routinely done in RNA-seq data analysis. Based on 300 simulation replicates, the empirical rejection rates of the Wald test for testing each predictor are reported in Table 1. NegBin regression has well controlled type I error rate for the null predictors age and gender. However, it has almost no power for detecting the treatment effect. The multinomial-logit model has seriously inflated type I error for the two null predictors age and gender. As a prelude, we also fit three other GLMs to the same data sets: Dirichlet-multinomial regression (DM), generalized Dirichletmultinomial (GDM) regression, and negative multinomial (NM) regression. Details of these models are given in Section 3. We find that GDM shows both well controlled type I error for age and gender and high power for detecting the treatment effect. Model selection criteria AIC (Akaike information criterion) and BIC (Bayesian information criterion) also indicate appropriateness of GDM. AIC/BIC of negative binomial regression is not listed because it uses sum of counts and is incomparable to the multivariate models. We remark that the generative model has a marginal negative binomial distribution and it has nothing to do with the GDM model. Success of GDM results from its ability to learn the complex correlation between counts.

\section{Models}

Table 2 lists four regression models for multivariate count responses. They impose different correlation structures on the counts. Except for the multinomial-logit model, none of the other three belongs to the natural exponential family. We denote the data by $\left(\boldsymbol{y}_{i}, \boldsymbol{x}_{i}\right), i=1, \ldots$, $n$, where $\boldsymbol{y}_{i} \in \mathbb{N}^{d}$ are $d$-dimensional count vectors and $\boldsymbol{x}_{i} \in \mathbb{R}^{p}$ are $p$-dimensional covariates. $\boldsymbol{Y}=\left(\boldsymbol{y}_{1}, \ldots, \boldsymbol{y}_{n}\right)^{\top} \in \mathbb{N}^{n \times d}$ and $\boldsymbol{X}=\left(\boldsymbol{x}_{1}, \ldots, \boldsymbol{x}_{n}\right)^{\top} \in \mathbb{R}^{n \times p}$ are called the response and design matrix respectively. For each model, we introduce the response distribution, propose a link function, and derive the score and information matrices (listed in Supplementary Materials S.2), which are essential for statistical estimation and inference. 


\section{Multinomial Regression (MN)}

To be self-contained, we start with the classical multinomial-logit model (McCullagh and Nelder, 1983). The response $\boldsymbol{y}$ is modeled as multinomial with $m=|\boldsymbol{y}|=\sum_{j=1}^{d} y_{j}$ trials and success probability parameter $\boldsymbol{p}=\left(p_{1}, \ldots, p_{d}\right), p_{j}>0, \sum_{j=1}^{d} p_{j}=1$. The probability mass function is

$$
f(\boldsymbol{y} \mid \boldsymbol{p})=\left(\begin{array}{c}
m \\
\boldsymbol{y}
\end{array}\right) \prod_{j=1}^{d} p_{j}^{y_{j}}
$$

It is well known that the multinomial distribution belongs to the natural exponential family. Parameter $\boldsymbol{p}$ is linked to the covariates $\boldsymbol{x} \in \mathbb{R}^{p}$ via the multinomial-Poisson transformation (Baker, 1994)

$$
p_{j}=\frac{e^{x^{\top} \beta_{j}}}{\sum_{j^{\prime}} e^{\boldsymbol{x}^{\top} \boldsymbol{\beta}_{j^{\prime}}}}, \quad j=1, \ldots, d
$$

where $\boldsymbol{\beta}_{1}, \ldots, \boldsymbol{\beta}_{d} \in \mathbb{R}^{p}$ are the regression coefficients. Because of the constraint $\Sigma_{j} p_{j}=1$, we set $\boldsymbol{\beta}_{d}=\mathbf{0}_{p}$ for identifiability and only estimate $\boldsymbol{\beta}_{1}, \ldots, \boldsymbol{\beta}_{d-1}$, which are collected in the regression coefficient matrix $\boldsymbol{B} \in \mathbb{R}^{p \times(d-1)}$. Log-likelihood of $n$ independent observations $\left(\boldsymbol{y}_{i}\right.$, $\left.\boldsymbol{x}_{i}\right)$ is

$$
\ell_{n}(\boldsymbol{B})=\sum_{i=1}^{n} \sum_{j=1}^{d} y_{i j}\left(\boldsymbol{x}_{i}^{\top} \boldsymbol{\beta}_{j}-\ln \sum_{j^{\prime}=1}^{d} e^{\boldsymbol{x}_{i}^{\top} \boldsymbol{\beta}_{j^{\prime}}}\right)+\sum_{i=1}^{n} \ln \left(\begin{array}{c}
m_{i} \\
\boldsymbol{y}_{i}
\end{array}\right) .
$$

The mapping $\left(\eta_{1}, \ldots, \eta_{d}\right)^{\top} \mapsto-\ln \Sigma_{j} e^{\eta_{j}}$ is concave; thus the log-likelihood function (1) is concave.

\section{Dirichlet-multinomial Regression (DM)}

Multinomial model is not sufficient when there is observed over-dispersion. Dirichletmultinomial distribution models the variation among the percentages $\boldsymbol{p}$ in the multinomial distribution by a Dirichlet distribution (Mosimann, 1962). The probability mass of a $d-$ category count vector $\boldsymbol{y}$ over $m=|\boldsymbol{y}|=\Sigma_{j} y_{j}$ trials under Dirichlet-multinomial with parameter $\boldsymbol{a}=\left(a_{1}, \ldots, a_{d}\right), a_{j}>0$, is 


$$
\begin{gathered}
f(\boldsymbol{y} \mid \boldsymbol{\alpha})=\int_{\Delta_{d}}\left(\begin{array}{c}
m \\
\boldsymbol{y}
\end{array}\right) \prod_{j} p_{j}^{y_{j}} \frac{\Gamma(|\boldsymbol{\alpha}|)}{\prod_{j} \Gamma\left(\alpha_{j}\right)} \prod_{j} p_{j}^{\alpha_{j}-1} d \boldsymbol{p} \\
=\left(\begin{array}{c}
m \\
\boldsymbol{y}
\end{array}\right) \prod_{j=1}^{d} \frac{\Gamma\left(\alpha_{j}+y_{j}\right)}{\Gamma\left(\alpha_{j}\right)} \frac{\Gamma\left(\sum_{j} \alpha_{j}\right)}{\Gamma\left(\sum_{j} \alpha_{j}+\sum_{j} y_{j}\right)} \\
=\left(\begin{array}{c}
m \\
\boldsymbol{y}
\end{array}\right) \frac{\prod_{j=1}^{d}\left(\alpha_{j}\right)_{\left(y_{j}\right)}}{(|\boldsymbol{\alpha}|)_{(m)}},
\end{gathered}
$$

where $(a)_{(k)}=a(a+1) \cdots(a+k-1)$ denotes the rising factorial and $|a|=\Sigma_{j} a_{j}$. Because the data $y_{j}$ and parameter $a_{j}$ are intertwined in the gamma terms and do not factorize, Dirichletmultinomial distribution does not belong to the natural exponential family. The first two moments of DM are

$$
\mathrm{E}(\boldsymbol{Y})=m \frac{\boldsymbol{\alpha}}{|\boldsymbol{\alpha}|}, \quad \operatorname{Cov}(\boldsymbol{Y})=m \frac{|\boldsymbol{\alpha}|+m}{|\boldsymbol{\alpha}|+1}\left[\operatorname{diag}\left(\frac{\boldsymbol{\alpha}}{|\boldsymbol{\alpha}|}\right)-\left(\frac{\boldsymbol{\alpha}}{|\boldsymbol{\alpha}|}\right)\left(\frac{\boldsymbol{\alpha}}{|\boldsymbol{\alpha}|}\right)^{\top}\right]
$$

It is clear that the counts are negatively correlated and the quantity $(|\boldsymbol{a}|+m) /(|\boldsymbol{a}|+1)$ measures over-dispersion. To incorporate covariates, the inverse link function $a_{j}=e^{X^{\top}} \beta_{j}$ relates the parameter $\boldsymbol{a}=\left(a_{1}, \ldots, a_{d}\right)$ of Dirichlet-multinomial distribution to the covariates $\boldsymbol{x}$. The $\log$-likelihood for $n$ independent data points $\left(\boldsymbol{y}_{i}, \boldsymbol{x}_{i}\right)$ takes the form

$$
\ell_{n}(\boldsymbol{B})=\sum_{i=1}^{n} \sum_{j=1}^{d} \sum_{k=0}^{y_{i j}-1} \ln \left(e^{\boldsymbol{x}_{i}^{\top} \boldsymbol{\beta}_{j}}+k\right)-\sum_{i=1}^{n} \sum_{k=0}^{m_{i}-1} \ln \left(\sum_{j=1}^{d} e^{\boldsymbol{x}_{i}^{\top} \boldsymbol{\beta}_{j}}+k\right)+\sum_{i=1}^{n} \ln \left(\begin{array}{c}
m_{i} \\
\boldsymbol{y}_{i}
\end{array}\right)
$$

where $\boldsymbol{B}=\left(\boldsymbol{\beta}_{1}, \ldots, \boldsymbol{\beta}_{d}\right) \in \mathbb{R}^{p \times d}$ collects all regression coefficients. The log-likelihood, as a difference of two concave terms, is not concave in general.

\section{Negative Multinomial Regression (NM)}

Negative correlation of the multinomial and Dirichlet-multinomial models prevents their use for positively correlated counts. The negative multinomial distribution provides a model for such data. The probability mass of a count vector $\boldsymbol{y} \in \mathbb{N}^{d}$ under a negative multinomial distribution with parameter $\left(p_{1}, \ldots, p_{d+1}, \beta\right), \sum_{j=1}^{d+1} p_{j}=1, p_{j}, \beta>0$, is

$$
f(\boldsymbol{y} \mid \boldsymbol{p}, \beta)=\left(\begin{array}{c}
\beta+m-1 \\
m
\end{array}\right)\left(\begin{array}{c}
m \\
\boldsymbol{y}
\end{array}\right) \prod_{j=1}^{d} p_{j}^{y_{j}} p_{d+1}^{B}=\frac{(\beta)_{(m)}}{m !}\left(\begin{array}{c}
m \\
\boldsymbol{y}
\end{array}\right) \prod_{j=1}^{d} p_{j}^{y_{j}} p_{d+1}^{\beta} .
$$

Parameter $\beta$ and data $m$ do not factorize; thus negative multinomial does not belong to the exponential family when $\beta$ is unknown. Denote $\boldsymbol{p}=\left(p_{1}, \ldots, p_{d}\right)$. Then the first two moments are

J Comput Graph Stat. Author manuscript; available in PMC 2018 February 16. 


$$
\mathrm{E}(\boldsymbol{Y})=\beta\left(\frac{\boldsymbol{p}}{p_{d+1}}\right), \quad \operatorname{Cov}(\boldsymbol{Y})=\beta\left[\operatorname{diag}\left(\frac{\boldsymbol{p}}{p_{d+1}}\right)+\left(\frac{\boldsymbol{p}}{p_{d+1}}\right)\left(\frac{\boldsymbol{p}}{p_{d+1}}\right)^{\top}\right],
$$

showing positive pairwise correlation between $Y_{j}$. We use the link functions

$$
p_{j}=\frac{e^{\boldsymbol{x}^{\top} \boldsymbol{\alpha}_{j}}}{1+\sum_{j=1}^{d} e^{\boldsymbol{x}^{\top} \boldsymbol{\alpha}_{j}}}, 1 \leq j \leq d, \quad p_{d+1}=\frac{1}{1+\sum_{j=1}^{d} e^{\boldsymbol{x}^{\top} \boldsymbol{\alpha}_{j}}}, \quad \beta=e^{\boldsymbol{x}^{\top} \boldsymbol{\beta}}
$$

to relate covariates $\boldsymbol{x} \in \mathbb{R}^{p}$ to distribution parameter $\left(p_{1}, \ldots, p_{d+1}, \boldsymbol{\beta}\right)$. Let $\boldsymbol{B}=\left(\boldsymbol{a}_{1}, \ldots, \boldsymbol{a}_{d}, \boldsymbol{\beta}\right)$ $\in \mathbb{R}^{p \times(d+1)}$ collect all the regression coefficients. Given $n$ independent data points $\left(\boldsymbol{y}_{i}, \boldsymbol{x}_{i}\right)$, the log-likelihood is

$\ell_{n}(\boldsymbol{B})=\sum_{i=1}^{n} \sum_{k=0}^{m_{i}-1} \ln \left(e^{\boldsymbol{x}_{i}^{\top} \boldsymbol{\beta}}+k\right)-\sum_{i=1}^{n}\left(e^{\boldsymbol{x}_{i}^{\top} \boldsymbol{\beta}}+m_{i}\right) \ln \left(\sum_{j=1}^{d} e^{\boldsymbol{x}_{i}^{\top} \boldsymbol{\alpha}_{j}+1}\right)+\sum_{i=1}^{n} \sum_{j=1}^{d} y_{i j} \boldsymbol{x}_{i}^{\top} \boldsymbol{\alpha}_{j}-\sum_{i=1}^{n} \sum_{j=1}^{d} \ln y_{i j} !$

When the over-dispersion parameter $\beta$ is not linked to covariates, the log-likelihood becomes

$\ell_{n}(\boldsymbol{B})=\sum_{i=1}^{n} \sum_{k=0}^{m_{i}-1} \ln (\beta+k)-\sum_{i=1}^{n}\left(\beta+m_{i}\right) \ln \left(\sum_{j=1}^{d} e^{\left.\boldsymbol{x}_{i}^{\top} \boldsymbol{\alpha}_{j}+1\right)}+\sum_{i=1}^{n} \sum_{j=1}^{d} y_{i j} \boldsymbol{x}_{i}^{\top} \boldsymbol{\alpha}_{j}-\sum_{i=1}^{n} \sum_{j=1}^{d} \ln y_{i j} !\right.$,

where $\boldsymbol{B}=\left(\boldsymbol{\alpha}_{1}^{T}, \ldots, \boldsymbol{\alpha}_{d}^{T}, \beta\right)^{T} \in \mathbb{R}^{p d+1}$. Neither log-likelihood (4) nor (5) is necessarily concave.

\section{Generalized Dirichlet-multinomial Regression (GDM)}

It is possible to relax the restrictions of pairwise negative correlation in $\mathrm{MN}$ and $\mathrm{DM}$ or pairwise positive correlation in NM by choosing a more flexible mixing distribution as a prior for the multinomial. Connor and Mosimann (1969) suggest a generalized Dirichletmultinomial distribution, which provides a flexible model for multivariate categorical responses with general correlation structure.

The probability mass of a count vector $\boldsymbol{y}$ over $m$ trials under the generalized Dirichletmultinomial model with parameter $(\boldsymbol{a}, \boldsymbol{\beta})=\left(a_{1}, \ldots, a_{d-1}, \beta_{1}, \ldots, \beta_{d-1}\right), a_{j}, \beta_{j}>0$, is 


$$
\begin{aligned}
f(\boldsymbol{y} \mid \boldsymbol{\alpha}, \boldsymbol{\beta})= & \left(\begin{array}{c}
m \\
\boldsymbol{y}
\end{array}\right) \prod_{j=1}^{d-1} \frac{\Gamma\left(\alpha_{j}+y_{j}\right)}{\Gamma\left(\alpha_{j}\right)} \frac{\Gamma\left(\beta_{j}+z_{j+1}\right)}{\Gamma\left(\beta_{j}\right)} \frac{\Gamma\left(\alpha_{j}+\beta_{j}\right)}{\Gamma\left(\alpha_{j}+\beta_{j}+z_{j}\right)} \\
& =\left(\begin{array}{c}
m \\
\boldsymbol{y}
\end{array}\right) \prod_{j=1}^{d-1} \frac{\left(\alpha_{j}\right)_{\left(y_{j}\right)}\left(\beta_{j}\right)_{\left(z_{j+1}\right)}}{\left(\alpha_{j}+\beta_{j}\right)_{\left(z_{j}\right)}}
\end{aligned}
$$

where $z_{j}=\sum_{k=j}^{d} y_{k}$. The Dirichlet-multinomial (DM) distribution is a special case of GDM by taking $\beta_{j}=a_{j+1}+\cdots+a_{d}$. GDM does not belong to the exponential family. The moments of the GDM are recorded in Lemma 1. A distinctive property of the GDM is that the correlations between counts can be simultaneously positive or negative, yielding the most modeling flexibility among the models in Table 2 . Below $(a)_{[k]}=a(a-1) \cdots(a-k+1)$ denote the falling factorial.

Lemma 1-The falling factorial moments of GDM are

$$
\mathrm{E} \prod_{j=1}^{d}\left(Y_{j}\right)_{\left[r_{j}\right]}=\mathrm{E} \prod_{j=1}^{d} Y_{j}\left(Y_{j}-1\right) \cdots\left(Y_{j}-r_{j}+1\right)=(m)_{\left[\sum_{j} r_{j}\right]} \prod_{j=1}^{d-1} \frac{\left(\alpha_{j}\right)_{\left(r_{j}\right)}\left(\beta_{j}\right)_{\left(\delta_{j+1}\right)}}{\left(\alpha_{j}+\beta_{j}\right)_{\left(\delta_{j}\right)}},
$$

where $\delta_{j}=r_{j}+\cdots+r_{d}$ for $j=1, \ldots, d$. Specifically the first two moments are

$$
\mathrm{E} Y_{j}=m\left\{\begin{array}{cc}
\frac{\alpha_{1}}{\alpha_{1}+\beta_{1}} & j=1 \\
\frac{\alpha_{j}}{\alpha_{j}+\beta_{j}} \prod_{k=1}^{j-1} \frac{\beta_{k}}{\alpha_{k}+\beta_{k}} & j=2, \ldots, d-1 \\
\prod_{k=1}^{d-1} \frac{\beta_{j}}{\alpha_{j}+\beta_{j}} & j=d
\end{array}\right.
$$

and

$$
\operatorname{Cov}\left(Y_{j}, Y_{j^{\prime}}\right)=m \frac{\alpha_{j^{\prime}}}{\alpha_{j^{\prime}}+\beta_{j^{\prime}}} \prod_{k=1}^{j^{\prime}-1} \frac{\beta_{k}}{\alpha_{k}+\beta_{k}} \times\left((m-1) \prod_{k=1}^{j-1} \frac{\beta_{k}+1}{\alpha_{k}+\beta_{k}+1} \frac{\alpha_{j}+1_{\left\{j=j^{\prime}\right\}}}{\alpha_{j}+\beta_{j}+1}-m \prod_{k=1}^{j-1} \frac{\beta_{k}}{\alpha_{k}+\beta_{k}} \frac{\alpha_{j}}{\alpha_{j}+\beta_{j}}+1_{\left\{j=j^{\prime}\right\}}\right)
$$

for $j \leq j^{\prime}$.

We employ the link functions

$$
\alpha_{j}=e^{\boldsymbol{x}^{\top} \boldsymbol{\alpha}_{j}}, \quad \beta_{j}=e^{\boldsymbol{x}^{\top} \boldsymbol{\beta}_{j}}, \quad 1 \leq j \leq d-1,
$$

to relate covariates $\boldsymbol{x}$ to distribution parameters $\left(a_{1}, \ldots, a_{d-1}, \beta_{1}, \ldots, \beta_{d-1}\right)$ of the GDM model. Here we slightly abuse notation and use scalar $a_{j}$ and $\beta_{j}$ to denote parameters of the GDM distribution. Boldfaced vectors $\boldsymbol{a}_{j}$ and $\boldsymbol{\beta}_{j}$, both of dimension $p$, denote regression 
coefficients. Let $\boldsymbol{B}=\left(\boldsymbol{a}_{1}, \ldots, \boldsymbol{a}_{d-1}, \boldsymbol{\beta}_{1}, \ldots, \boldsymbol{\beta}_{d-1}\right) \in \mathbb{R}^{p \times 2(d-1)}$ collect all the regression coefficients. Given $n$ independent data points $\left(\boldsymbol{y}_{i}, \boldsymbol{x}_{i}\right)$, the log-likelihood is

$$
\boldsymbol{\ell}_{n}(\boldsymbol{B})=\sum_{i=1}^{n} \sum_{j=1}^{d-1}\left[\sum_{k=0}^{y_{i j}-1} \ln \left(e^{\boldsymbol{x}_{i}^{\top} \boldsymbol{\alpha}_{j}}+k\right)+\sum_{k=0}^{z_{i, j+1}-1} \ln \left(e^{\boldsymbol{x}_{i}^{\top} \boldsymbol{\beta}_{j}}+k\right)-\sum_{k=0}^{z_{i j}-1} \ln \left(e^{\boldsymbol{x}_{i}^{\top} \boldsymbol{\alpha}_{j}}+e^{\boldsymbol{x}_{i}^{\top} \boldsymbol{\beta}_{j}}+k\right)\right]+\sum_{i=1}^{n} \ln \left(\begin{array}{c}
m_{i} \\
\boldsymbol{y}_{i}
\end{array}\right)
$$

which is not concave in general.

\section{Model Choice}

Multinomial is a limiting case of Dirichlet-multinomial by taking $\boldsymbol{a} /|\boldsymbol{a}| \rightarrow \boldsymbol{p}$ as $|\boldsymbol{a}| \rightarrow \infty$. Dirichlet-multinomial is a special case of generalized Dirichlet-multinomial by taking $\beta_{j}=$ $a_{j+1}+\cdots+a_{d}$. Therefore for distribution fitting, standard tests such as the likelihood ratio test (LRT) help choose the best one among the three nested models: MN $\subset$ DM $\subset$ GDM.

However, this nesting structure is lost in regression models. For instance, in presence of predictors, multinomial regression is not a sub-model of the Dirichlet-multinomial regression model, and the latter is not a special case of the generalized Dirichlet-multinomial (GDM) regression model. Information criteria such as the AIC and BIC can be used to determine a best regression model for the data.

\section{Estimation}

In this section we consider the MLE when sample size is greater than the number of parameters. In Section 6 we consider regularized estimation that is useful for model selection. MLE for the DM, NM and GDM models is nontrivial as they do not belong to the natural exponential family and the classical IRWLS (iteratively re-weighted least squares) machinery is difficult to apply, as explained in Section 4.1.

\subsection{Difficulties}

We illustrate the difficulties using Dirichlet-multinomial (DM) regression as an example. Given iid data $\left(\boldsymbol{y}_{i}, \boldsymbol{x}_{i}\right)$, let $\boldsymbol{X}=\left(\boldsymbol{x}_{1}, \ldots, \boldsymbol{x}_{n}\right)^{\top} \in \mathbb{R}^{n \times p}$ be the design matrix and $\boldsymbol{A}=\left(a_{i j}\right) \in$ $\mathbb{R}^{n \times d}$, where $\alpha_{i j}=e^{\boldsymbol{x}_{i}^{\top} \boldsymbol{\beta}_{j}}$, be the matrix of distribution parameters at each observation. The observed information matrix is

$-d^{2} \ell_{n}(\boldsymbol{B})=\left(\boldsymbol{I}_{d} \otimes \boldsymbol{X}\right)^{\top} \cdot \operatorname{diag}[\operatorname{vec}(\operatorname{diag}(\boldsymbol{v}) \boldsymbol{A}-\boldsymbol{K})] \cdot\left(\boldsymbol{I}_{d} \otimes \boldsymbol{X}\right)-\left(\boldsymbol{A}^{\top} \odot \boldsymbol{X}^{\top}\right) \operatorname{diag}(\boldsymbol{w})\left(\boldsymbol{A}^{\top} \odot \boldsymbol{X}^{\top}\right)^{\top}$,

where $\boldsymbol{v} \in \mathbb{R}^{n}$ has entries $v_{i}=\sum_{k=1}^{m_{i}-1}\left(\left|\boldsymbol{\alpha}_{i}\right|+k\right)^{-1}, \boldsymbol{w} \in \mathbb{R}^{n}$ has entries

$w_{i}=\sum_{k=0}^{m_{i}-1}\left(\left|\boldsymbol{\alpha}_{i}\right|+k\right)^{-2}$, and $\boldsymbol{K}=\left(k_{i j}\right) \in \mathbb{R}^{n \times d}$ has entries 


$$
k_{i j}=\sum_{k=0}^{y_{i j}-1}\left(\frac{\alpha_{i j}}{\alpha_{i j}+k}\right)\left(\frac{k}{\alpha_{i j}+k}\right) .
$$

Here $\otimes$ denotes the Kronecker product and $\odot$ denotes the Khatri-Rao product. See Supplementary Materials S.2.2 for the derivation.

Traditional optimization methods encounter difficulties when maximizing the DM loglikelihood. The Newton-Raphson method can be unstable because of the non-concavity of the log-likelihood (3). The observed information matrix $-d^{2} l_{n}(\boldsymbol{B})$ is not necessarily positive definite and the Newton iterates may diverge. This issue is serious in regression problems because often there is no good starting point available. The Fisher scoring algorithm, also known as the iteratively re-weighted least squares (IRWLS), replaces the observed information matrix by its expectation, i.e., the Fisher information matrix $E\left[-d^{2} \ell_{n}(\boldsymbol{B})\right]$. The Fisher information matrix is always positive semidefinite and, combined with line search, is guaranteed to generate nondecreasing iterates. However, evaluation of the Fisher information matrix involves computing

$$
\mathrm{E}\left(k_{i j}\right)=\mathrm{E}\left[\sum_{k=0}^{y_{i j}-1}\left(\frac{\alpha_{i j}}{\alpha_{i j}+k}\right)\left(\frac{k}{\alpha_{i j}+k}\right)\right]=\sum_{k=0}^{m_{i}-1}\left(\frac{\alpha_{i j}}{\alpha_{i j}+k}\right)\left(\frac{k}{\alpha_{i j}+k}\right) \mathrm{P}\left(Y_{i j}>k\right),
$$

where $\left(Y_{i 1}, \ldots, Y_{i d}\right)$ is a Dirichlet-multinomial random vector with parameter $\left(a_{i 1}, \ldots, a_{i d}\right)$ and batch size $m_{i}$. Marginal Beta-binomial tail probabilities $\mathrm{P}\left(Y_{i j}>k\right)$ have to be evaluated for each combination of $i \in\{1, \ldots, n\}, j \in\{1, \ldots, d\}$, and $k \in\left\{0, \ldots, m_{i}-1\right\}$, making Fisher scoring method computationally expensive for even moderate sample size $n$ and $d$. Even when the information matrix is positive definite and can be evaluated, a $p(d-1)$ by $p(d$ - 1) linear system needs to be solved in each Newton or scoring iteration. Finally, QuasiNewton methods such as BFGS updates (Nocedal and Wright, 2006) may alleviate some of the issues but suffer from slow convergence and instability in many examples.

Table 3 reports results from a simple numerical experiment. DM data are generated with $d=$ $3,15,20,30$ response categories, $p=6$ predictors, and sample size $n=200$. The true parameter value is set at $\boldsymbol{B}=3 \times \mathbf{1}_{p \times d}$. Entries of the design matrix $\boldsymbol{X}$ are drawn from independent standard normals. For each $d, 100$ replicates are simulated. For each replicate, we use the nlminb function in R to fit DM regression using Newton (with analytical gradient and Hessian) and quasi-Newton (BFGS with analytical gradient) methods. The starting point for each run is set at $\boldsymbol{B}^{(0)}=\mathbf{0}_{p \times d}$. For small dimensional problem $d=3$, Newton method converges in $72 \%$ of replicates. As $d$ increases, Newton's method fails more and more often. The Quasi-Newton method apparently suffers from both instability and slow convergence.

The same difficulties, namely instability, high cost of evaluating Fisher information matrix, and high dimensionality of parameters, beset the MLE for the negative multinomial (NM) and generalized Dirichlet-multinomial (GDM) regressions. 


\subsection{MLE via IRPR}

We propose a unifying framework, the iteratively reweighted Poisson regression (IRPR), for the MLE of the four regression models. The plain version of the IRPR scheme is summarized in Algorithm 1. At each iteration, we update some working responses $y_{i j}^{(t)}$ and weights $w_{i j}^{(t)}$ and then perform $d_{e}$ weighted Poisson regressions to update the regression parameters. Here $d_{e}=d-1$ for MN, $d$ for DM, 2( $\left.d-1\right)$ for GDM, and $d+1$ for NM. Therefore IRPR is extremely simple to implement using existing software such as R and Matlab with a sound Poisson regression solver. Specific choice of the working responses and weights for each model is listed below.

\section{Algorithm 1}

Iterative reweighted Poisson regression (IRPR) scheme for MLE. Working responses $y_{i j}^{(t)}$ and weights $w_{i j}^{(t)}$ for each model are specified in Section 4.2.

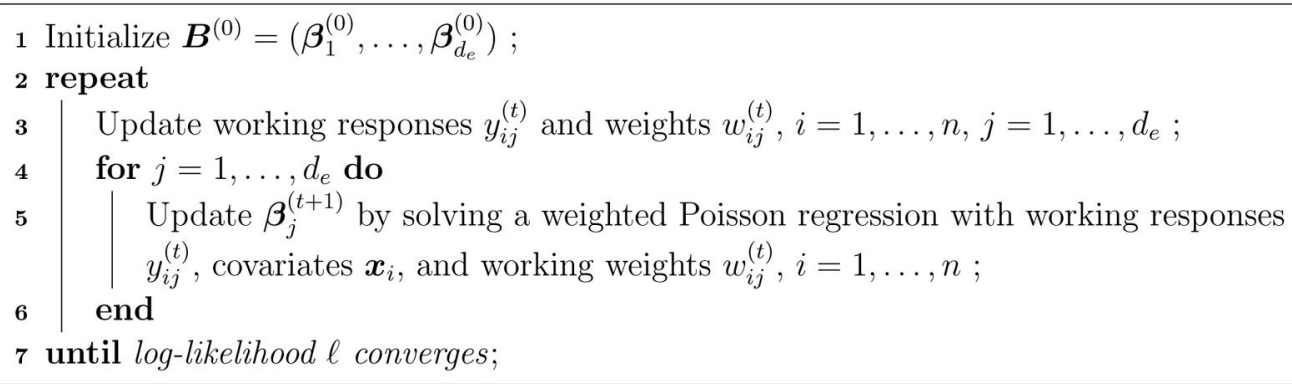

- $\quad$ Multinomial-Logit Regression (MN).

Given $\boldsymbol{B}^{(t)}=\left(\boldsymbol{\beta}_{1}^{(t)}, \ldots, \boldsymbol{\beta}_{d-1}^{(t)}\right)$, we update $\boldsymbol{\beta}_{j}, j=1, \ldots, \boldsymbol{d}-1$, by solving a Poisson regression with working weights and responses

$$
w_{i j}^{(t)}=\frac{m_{i}}{\sum_{j^{\prime}=1}^{d} e^{\boldsymbol{x}_{i}^{\top} \boldsymbol{\beta}_{j^{\prime}}^{(t)}}}, \quad y_{i j}^{(t)}=\frac{y_{i j}}{w_{i j}^{(t)}} .
$$

- $\quad$ Dirichlet-multinomial Regression (DM).

Given $\boldsymbol{B}^{(t)}=\left(\boldsymbol{\beta}_{1}^{(t)}, \ldots, \boldsymbol{\beta}_{d}^{(t)}\right)$, we update $\boldsymbol{\beta}_{j}, j=1, \ldots, d$, by solving a Poisson regression with working weights and responses

$$
w_{i j}^{(t)}=\sum_{k=0}^{m_{i}-1}\left(\sum_{j^{\prime}} e^{\boldsymbol{x}_{i}^{\top} \boldsymbol{\beta}_{j^{\prime}}^{(t)}}+k\right)^{-1}, \quad y_{i j}^{(t)}=\left(w_{i j}^{(t)}\right)^{-1}\left(\sum_{k=0}^{y_{i j}-1} \frac{e^{\boldsymbol{x}_{i}^{\top} \boldsymbol{\beta}_{j}^{(t)}}}{e^{\boldsymbol{x}_{i}^{\top} \boldsymbol{\beta}_{j}^{(t)}+k}}\right) .
$$

- Generalized Dirichlet-multinomial Regression (GDM). 
Given $\boldsymbol{B}^{(t)}=\left(\boldsymbol{\alpha}_{1}^{(t)}, \ldots, \boldsymbol{\alpha}_{d-1}^{(t)}, \boldsymbol{\beta}_{1}^{(t)}, \ldots, \boldsymbol{\beta}_{d-1}^{(t)}\right)$, we update $\boldsymbol{a}_{j}, j=1, \ldots, d-1$, by solving a weighted Poisson regression with working weights and responses

$$
w_{i j}^{(t)}=\sum_{k=0}^{z_{i j}-1}\left(e^{\boldsymbol{x}_{i}^{\top} \boldsymbol{\alpha}_{j}^{(t)}}+e^{\boldsymbol{x}_{i}^{\top} \boldsymbol{\beta}_{j}^{(t)}}+k\right)^{-1}, \quad y_{i j}^{(t)}=\left(w_{i j}^{(t)}\right)^{-1}\left(\sum_{k=0}^{y_{i j}-1} \frac{e^{\boldsymbol{x}_{i}^{\top} \boldsymbol{\alpha}_{j}^{(t)}}}{e^{\boldsymbol{x}_{i}^{\top} \boldsymbol{\alpha}_{j}^{(t)}}+k}\right),
$$

and update $\boldsymbol{\beta}_{j}, j=1, \ldots, d-1$, by solving a weighted Poisson regression with working weights and responses

$$
w_{i j}^{(t)}=\sum_{k=0}^{z_{i j}-1}\left(e^{\boldsymbol{x}_{i}^{\top} \boldsymbol{\alpha}_{j}^{(t)}}+e^{\boldsymbol{x}_{i}^{\top} \boldsymbol{\beta}_{j}^{(t)}}+k\right)^{-1}, \quad y_{i j}^{(t)}=\left(w_{i j}^{(t)}\right)^{-1}\left(\sum_{k=0}^{y_{i, j+1}-1} \frac{e^{\boldsymbol{x}_{i}^{\top} \boldsymbol{\beta}_{j}^{(t)}}}{e^{\boldsymbol{x}_{i}^{\top} \boldsymbol{\beta}_{j}^{(t)}}+k}\right) .
$$

\section{- $\quad$ Negative multinomial Regression (NM)}

Given $\boldsymbol{B}^{(t)}=\left(\boldsymbol{\alpha}_{1}^{(t)}, \ldots, \boldsymbol{\alpha}_{d}^{(t)}, \boldsymbol{\beta}^{(t)}\right)$, we update $\boldsymbol{\beta}$ by solving a weighted Poisson regression with working weights and responses

$$
w_{i}^{(t)}=\ln \left(\sum_{j=1}^{d} e^{\boldsymbol{x}_{i}^{\top} \boldsymbol{\alpha}_{j}^{(t)}+1}\right), \quad y_{i}^{(t)}=\left(w_{i}^{(t)}\right)^{-1}\left(\sum_{k=0}^{m_{i}-1} \frac{e^{\boldsymbol{x}_{i}^{\top} \boldsymbol{\beta}^{(t)}}}{e^{\boldsymbol{x}_{i}^{\top} \boldsymbol{\beta}^{(t)}}+k}\right) .
$$

Updating $\boldsymbol{a}_{j}, j=1, \ldots, d$, is a weighted Poisson regression with working weights and responses

$$
w_{i j}^{(t)}=\frac{e^{\boldsymbol{x}_{i}^{\top} \beta^{(t+1)}}+m_{i}}{\sum_{j=1}^{d} e^{\boldsymbol{x}_{i}^{\top} \boldsymbol{\alpha}_{j}^{(t)}}+1}, \quad y_{i j}^{(t)}=\frac{y_{i j}}{w_{i j}^{(t)}}
$$

IRPR is derived in Supplementary Materials S.3. Most importantly, IRPR iterations always increase the log-likelihood and thus enjoy superior stability.

Lemma 2 (Monotonicity)—The IRPR algorithmic iterates $\boldsymbol{B}^{(t)}$ satisfy $\ell\left(\boldsymbol{B}^{(t+1)}\right) \geq \ell\left(\boldsymbol{B}^{(t)}\right)$ for $t \geq 0$.

Monotonicity and simplicity of IRPR are reminiscent of the celebrated expectationmaximization (EM) algorithm (Dempster et al., 1977). Derivation of the IRPR hinges upon the minorization-maximization (MM) principle (Lange et al., 2000), a powerful generalization of the EM algorithm. The same principle has been successfully applied to distribution fitting (Zhou and Lange, 2010; Zhou and Zhang, 2012). Monotonicity of the algorithm does not guarantee the convergence of the iterates. The following proposition specifies conditions for the global convergence of the IPRP algorithm.

Proposition 1-Assume that (i) the design matrix $\boldsymbol{X}$ has full column rank, (ii) the loglikelihood $\ell$ is bounded above, (iii) the set $\left\{\boldsymbol{B}: \ell(\boldsymbol{B}) \geq \ell\left(\boldsymbol{B}^{(0)}\right)\right\}$ is compact, and (iv) the set of 
stationary points of $\ell$ are isolated. Then the IRPR iterates $\boldsymbol{B}^{(t)}$ converge to a stationary point of $\ell$

In practice, EM and MM algorithms may suffer from slow convergence. Their convergence rate is linear at best. We combine stability of IRPR and fast convergence of Newton method by using a mix-and-match strategy. During each iteration, we choose either the IRPR or the Newton update that yields a higher log-likelihood. This strategy works very well in practice, as exemplified by the exceptional stability and competitive run times in Table 3. Another pleasant observation is that the $d_{e}$ Poisson regressions within each iteration are separated, making IRPR amenable to parallel computing. This is a common feature of many EM or MM algorithms that are able to divide a high-dimensional optimization problem into many small ones (Zhou et al., 2010).

\section{Testing}

Scientific applications often involve testing the significance of covariate(s). Although the regression models in Table 2, except for the multinomial, do not belong to the exponential family, they are regular in many statistical senses as their densities are quadratically mean differentiable (qmd) (Lehmann and Romano, 2005). Hence the standard asymptotic tests (LRT, score, Wald) apply.

With $p$ covariates, each covariate involves $d_{e}$ regression parameters, leading to a total of $p d_{e}$ parameters. The likelihood ratio test (LRT) for two nested models is asymptotically distributed as a chi-square distribution with $d_{e} \Delta p$ degrees of freedom, where $\Delta p$ is the difference in the numbers of covariates. To apply the LRT, optimizations have to be performed under the null and alternative hypotheses separately. The score test avoids computing the MLE at the alternative hypothesis and the Wald test avoids optimization at the null hypothesis.

\section{Regularization}

The asymptotics fail when the sample size $n$ is only moderately larger than or even less than the number of regression parameters $p d_{e}$. In such cases, regularization is a powerful tool for reducing the variance of estimate and improving its mean squared error. In general we consider regularized problem

$$
\min _{\boldsymbol{B}} h(\boldsymbol{B})=-\ell(\boldsymbol{B})+J(\boldsymbol{B}),
$$

where $\ell$ is the $\log$-likelihood function and $J$ is a regularization functional. Choice of $J$ depends on specific applications.

- $\quad$ Different covariates may be associated with different category. Sparsity in terms of $\|\operatorname{vec} \boldsymbol{B}\|_{0}$ is sought in this situation. For instance, in a sparse Dirichletmultinomial regression model proposed by Chen and Li (2013), $\beta_{k j}$ being non- 
zero indicates association of predictor $k$ with bacterial taxon $j$. In general, elementwise shrinkage and sparsity can be achieved by the regularization term

$$
J(\boldsymbol{B})=\sum_{k \in \mathscr{S}_{j=1}} \sum_{\eta} P_{\eta}\left(\left|\beta_{k j}\right|, \lambda\right)
$$

where the set $\mathscr{S}$ indexes the covariates subject to regularization, $P_{\eta}(|x|, \lambda)$ is a scalar penalty function, $\lambda$ is the penalty tuning parameter, and $\eta$ is an index for the penalty family. Widely used penalties include: power family (Frank and Friedman, 1993), where $P_{\eta}(|x|, \lambda)=\lambda|x|^{\eta}, \eta \in(0,2]$, and in particular lasso (Tibshirani, 1996) $(\eta=1)$ and ridge $(\eta=2)$; elastic net (Zou and Hastie, 2005), where $P_{\eta}(|x|, \lambda)=\lambda\left[(\eta-1) x^{2} / 2+(2-\eta)|x|\right], \eta \in[1,2]$; SCAD (Fan and Li, 2001), where $\partial / \partial|x| P_{\eta}(|x|, \lambda)=\lambda\left\{1_{\{|x| \leq \lambda\}}+\left[(\text { \&eegr; } \lambda-|x|)_{+} /(\eta-1)\right.\right.$

$\left.\lambda] 1_{\{|x|>\lambda\}}\right\}, \eta>2$; and MC+ penalty (Zhang, 2010), where $P_{\eta}(|x|, \lambda)=\{\lambda|x|-$ $\left.x^{2} /(2 \eta)\right\} 1_{\{|x|<\eta \lambda\}}+0.5 \lambda^{2} \eta 1_{\{|x| \geq \eta \lambda\}}$, among many others.

- $\quad$ Predictor selection can be achieved by the group penalty (Yuan and Lin, 2006; Meier et al., 2008)

$$
J(\boldsymbol{B})=\lambda \sum_{k \in \mathscr{S}}\left\|\boldsymbol{\beta}_{[k]}\right\|_{2}
$$

where $\lambda$ is the penalty tuning constant, $\boldsymbol{\beta}_{[k]}$ is the vector of regression coefficients associated with the $k$-th covariate, and $\|v\|_{2}$ is the $\ell_{2}$ norm of a vector $\boldsymbol{v}$. In other words, $\boldsymbol{\beta}_{[k]}$ is the $k$-th row of the regression parameter matrix $\boldsymbol{B} \in$ $\mathbb{R}^{p \times d_{e}}$.

- Sparsity at both the predictor level and within predictors can be achieved by the $\ell_{2,1}$ penalty (Zhao et al., 2009)

$$
J(\boldsymbol{B})=\lambda \sum_{k \in \mathscr{S}}\left(\sum_{j=1}^{d_{e}}\left|\beta_{k j}\right|\right)^{1 / 2} .
$$

Shrinkage and sparsity in terms of the rank of $\boldsymbol{B}$ is achieved by regularization term

$$
J(\boldsymbol{B})=\lambda\|\boldsymbol{B}\|_{*}=\lambda \sum_{i} \sigma_{i}(\boldsymbol{B})
$$

where the nuclear norm $\|\boldsymbol{B}\|_{*}=\Sigma_{j} \sigma_{j}(\boldsymbol{B})$, and $\sigma_{j}(\boldsymbol{B})$ 's are the singular values of the matrix $\boldsymbol{B}$. The nuclear norm $\|\boldsymbol{B}\|_{*}$ is a suitable measure of the 'size' of a matrix parameter, and is a convex relaxation of $\operatorname{rank}(\boldsymbol{B})=\|\sigma(\boldsymbol{B})\|_{0}$. This extends the reduced rank multivariate linear regression (Yuan et al., 2007) to multivariate GLM. 
The non-smooth minimization problem (8) is nontrivial. The MM principle underlying the IRPR algorithm in Section 4 is able to separate the columns of parameter matrix $\boldsymbol{B}$. Unfortunately both the group and nuclear norm regularization terms are not separable in columns. The success of the coordinate descent algorithm, which is efficient for $\ell_{1}$ regularization in univariate GLM models (Friedman et al., 2010), suggests the block descent algorithm for minimizing (8). However, updating each block is a possibly nonconvex problem and nontrivial.

We use the accelerated proximal gradient method that has been successful in solving various regularization problems (Beck and Teboulle, 2009). The accelerated proximal gradient algorithm as summarized in Algorithm 2 consists of two steps per iteration: (a) predicting a search point $\boldsymbol{S}$ based on the previous two iterates (line 11) and (b) performing gradient descent from the search point $S$, possibly with a line search (lines 3-7). We first describe step (b). The gradient descent step effectively minimizes the surrogate function

$$
\begin{aligned}
g\left(\boldsymbol{B} \mid \boldsymbol{S}^{(t)}, \delta\right) & =-\ell\left(\boldsymbol{S}^{(t)}\right)-\left\langle\nabla \ell\left(\boldsymbol{S}^{(t)}\right), \boldsymbol{B}-\boldsymbol{S}^{(t)}\right\rangle+\frac{1}{2 \delta}\left\|\boldsymbol{B}-\boldsymbol{S}^{(t)}\right\|_{\mathrm{F}}^{2}+J(\boldsymbol{B}) \\
& =\frac{1}{2 \delta}\left\|\boldsymbol{B}-\left[\boldsymbol{S}^{(t)}+\delta \nabla \ell\left(\boldsymbol{S}^{(t)}\right)\right]\right\|_{\mathrm{F}}^{2}+J(\boldsymbol{B})+c^{(t)},
\end{aligned}
$$

where the constant $c^{(t)}$ collects terms irrelevant to the optimization. Here we abuse the notation to use $\left.\nabla \ell S^{(t)}\right) \in \mathbb{R}^{p \times d_{e}}$ to denote the matrix of first derivatives $\partial \ell / \partial s_{k j}^{(t)}$. These gradients are given in Propositions S.1-S.3 of Supplementary Materials as the score functions for the models considered. The ridge term $(2 \delta)^{-1}\left\|\boldsymbol{B}-\boldsymbol{S}^{(t)}\right\|_{\mathrm{F}}^{2}$ in the surrogate function (9) shrinks the next iterate towards $S^{(t)}$, which is desirable since the first order approximation is good only within the neighborhood of current search point. Minimizing the surrogate function $g\left(\boldsymbol{B} \mid \boldsymbol{S}^{(t)}, \delta\right)$ is achieved by simple thresholding. Let $\left.\boldsymbol{A}_{\text {temp }}=\boldsymbol{S}^{(t)}+\delta \nabla \ell \boldsymbol{S}^{(t)}\right)$ be the intermediate matrix with rows $\boldsymbol{a}_{[k]}^{(t)}$. The minimizer of $g$, denoted by $\boldsymbol{B}_{\text {temp }}$, for various $J$ are listed below (line 5). 


\section{Algorithm 2}

Accelerated proximal gradient method for regularized estimation (8).

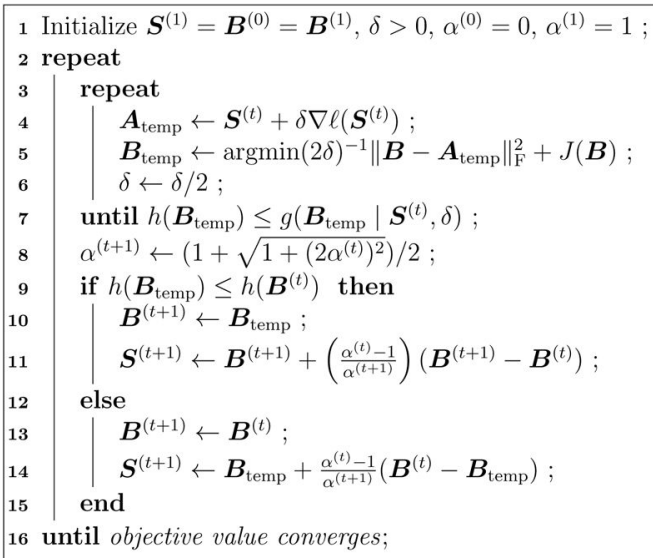

- $\quad$ Lasso penalty $\boldsymbol{J}(\boldsymbol{B})=\lambda\|\operatorname{vec}(\boldsymbol{B})\|_{1} . \boldsymbol{B}_{\text {temp }}$ has entries $\left(1-\delta \lambda / \mid a_{i j}\right)_{+} a_{i j}$.

- Group penalty $\boldsymbol{J}(\boldsymbol{B})=\lambda \Sigma_{k}\left\|\boldsymbol{\beta}_{[k]}\right\|_{2}$. The rows of $\boldsymbol{B}_{\text {temp }}$ are given by $(1-\delta \lambda / \|$ $\left.\boldsymbol{a}_{[k]} \|_{2}\right)_{+} \boldsymbol{a}_{[k]} \cdot$

- $\quad \ell_{2,1}$ penalty does not have analytic solution for the minimizer $g$. $\boldsymbol{B}_{\text {temp }}$ can be solved by weighted lasso regression (Xu et al., 2010).

- $\quad$ Nuclear norm $J(B)=\lambda\|\boldsymbol{B}\| *$. Suppose $\boldsymbol{A}_{\text {temp }}$ admits singular value decomposition $\boldsymbol{U} \operatorname{diag}(\boldsymbol{a}) \boldsymbol{V}^{\top}$. Then $\boldsymbol{B}_{\text {temp }}=\boldsymbol{U} \operatorname{diag}\left[(\boldsymbol{a}-\delta \boldsymbol{\lambda})_{+}\right] \boldsymbol{V}$.

Suppose the loss $-\mathscr{C}(\boldsymbol{B})$ has gradient Lipschitz constant $\mathscr{L}$, i.e., $\left\|\nabla \ell\left(\boldsymbol{B}_{1}\right)-\nabla \ell\left(\boldsymbol{B}_{2}\right)\right\| \leq \mathscr{L} \| \boldsymbol{B}_{1}-$ $\boldsymbol{B}_{2} \|_{\mathrm{F}}$ for all $\boldsymbol{B}_{1}, \boldsymbol{B}_{2}$. Then we can fix $\delta=\mathscr{L}^{-1}$ and the line search described in Algorithm 2 terminates in a single step. Using a larger $\delta$ leads to a bigger gradient descent step (line 4), which sometimes must be contracted to send the penalized loss downhill.

In step (a) of Algorithm 2. The search point $S$ is found by an extrapolation based on the previous two iterates $\boldsymbol{B}^{(t)}$ and $\boldsymbol{B}^{(t-1)}$. This trick accelerates ordinary gradient descent by making this extrapolation. Without extrapolation, Nesterov's method collapses to a gradient method with the slow non-asymptotic convergence rate of $O\left(k^{-1}\right)$ rather than $O\left(k^{-2}\right)$.

Assume the loss $-\ell$ has gradient Lipschitz constant $\mathscr{L}(\mathcal{C})$. For convex loss such as in the multinomial-logit model, if the global minimum of the penalized loss $h(\boldsymbol{B})$ occurs at the point $\boldsymbol{B}^{*}$, then the following non-asymptotic bound for the convergence of the objective values

$$
h\left(\boldsymbol{B}^{(t)}\right)-h\left(\boldsymbol{B}^{*}\right) \leq \frac{4 \mathscr{L}(f)\left\|\boldsymbol{B}^{(0)}-\boldsymbol{B}^{*}\right\|_{\mathrm{F}}^{2}}{(t+1)^{2}}
$$


applies (Beck and Teboulle, 2009). For non-convex losses such as in the DM, NM and GDM regressions, convergence theory is hard. In general, it is only guaranteed that $\left\|\boldsymbol{B}^{(t+1)}-\boldsymbol{B}^{(t)}\right\|_{\mathrm{F}}$ converges to 0 . In practice the algorithm almost always converges to at least a local minimum of the objective function.

\section{Numerical Examples}

We conducted extensive simulation studies to assess the finite sample performance of four regression models. Specifically we demonstrate that misspecification of the model can cause serious errors in hypothesis testing and variable selection. This is of practical importance as practitioners routinely rely on the multinomial-logit $(\mathrm{MN})$ model to analyze data with multiple categorical responses.

\subsection{Hypothesis Testing}

We generate polytomous responses from each of the four models, MN, DM, GDM and NM, and then fit the data with all four regression models.

In the generative model, there are $d=5$ categories and $p=6$ predictors. The first three predictors have nonzero effects size $a_{0}$ and the last three are null predictors. Therefore the true parameter matrix is $\boldsymbol{B}=\left[\alpha_{0}, \alpha_{0}, \alpha_{0}, 0,0,0\right]^{\top} \mathbf{1}_{d_{e}}^{\top} \in \mathbb{R}^{p \times d_{e}}$. The number of parameters to estimate is $24,30,48,31$ for MN, DM, GDM and NM respectively. Entries of the covariate matrix $\boldsymbol{X}$ are generated from independent standard normal. We vary the effect size $a_{0}$ at values $0,0.05,0.1,0.5,1,2$, and 5 and the sample size $n$ at 50, 100, 200 and 500. The batch size $m_{i}$ of MN, DM, GDM response vectors are generated from Binomial $(200,0.8)$. We simulate 300 replicates at each combination of effect size and sample size. Empirical type I error and power of the Wald test for testing the significance of the first predictor are reported.

Table 4 shows the results when responses are generated from the GDM model. The fact that using a wrong model, MN, DM or NM in this case, causes highly inflated type I error is cautionary, as practitioners routinely rely on the multinomial-logit model to analyze count data. Similar patterns are observed when the responses are generated from the MN, DM or NM models. Their results are presented in Tables $1-3$ of Supplementary Materials.

\subsection{Variable Selection by Regularization}

The simulation design for sparse regression is similar to the previous section, except that the response matrix has $d=10$ categories and there are $p=100$ predictors. Only the first 5 predictors are associated with the responses with effect size $a_{0} \in\{0.1,0.3,0.5,0.8,1\}$. Sample sizes are $n=25,50,75,100.300$ replicates are simulated at each combination of effect size and sample size. For each data replicate, we perform predictor selection by fitting the group penalized estimation.

The variable selection performance is summarized by the receiver operating characteristic (ROC) curves that result from the solution path. At each tuning parameter value $\lambda$, we calculate the true positive rate (TPr) and false positive rate (FPr) of the regularized estimate $\hat{\boldsymbol{B}}(\lambda)$ : 


$$
\begin{gathered}
\text { True positive rate }(\mathrm{TPr})=\frac{\# \text { true positives }}{\text { False positive rate }(\mathrm{FPr})}=\frac{\text { \# true positive+\# false negative }}{\# \text { false positive }} \\
\text { F false positive }+\# \text { true negative }
\end{gathered}
$$

At $\lambda=\infty, \mathrm{TPr}$ and FPr are 0 . Both increase as $\lambda$ decreases, approaching 1 at $\lambda=0$. Thus each solution path produces an ROC curve. For each regression model, the 300 ROC curves is summarized by an average ROC curve obtained from fitting an isotonic regression.

Figure 2 displays the summary ROC curves of the four regression models, when the responses are generated from the generalized Dirichlet-multinomial distribution (GDM). GDM regression demonstrates the best variable selection performance, followed by the DM regression. The multinomial (MN) and negative multinomial (NM) model have little discriminatory power at various effect sizes and sample sizes. To appreciate the Monte carlo errors, we also calculate the area under curve (AUC) of the ROC curve from each replicate. Larger AUC indicates better variable selection performance. The box plots of AUCs are displayed in Figure 3. Again the correct model GDM shows superior performance, while the $\mathrm{MN}$ and NM models are no better than random guess.

Supplementary Materials contain the corresponding summery ROC curves and box plots for AUCs when data were generated from multinomial (MN), Dirichlet-multinomial (DM), and negative multinomial (NM) models. In general we observe a similar pattern: using an incorrectly specified model leads to poor performance in variable selection.

\subsection{Real Data}

We apply the regression models to an RNA-seq data set of 60 HapMap CEU samples (Montgomery et al., 2010). Genotype data are also available for these samples and we aim to assess the association between the genotype of each SNP (Single Nucleotide Polymorphism) with the counts of multiple exon sets of each gene.

We demonstrate results by gene ST13P6 (suppression of tumorigenicity 13 pseudogene 6), which has been related to B-Cell leukemia, multiple myeloma, and prostate cancer in previous studies (Sossey-Alaoui et al., 2002). ST13P6 has 3 exon sets and is located on chromosome 8. Expression counts of the 3 exon sets are regressed on covariates, namely three principle components, $\log$ (TotalReads), and each of the 45,587 SNPs on chromosome 8 , using the four models in Table 2. The total expression counts are also regressed on the same covariates using the negative binomial (NegBin) model. Thus each model generates 45,587 p-values, which are summarized by the QQ plot and Manhattan plot. In reality association of expression levels and SNPs is rare. Therefore we expect none or at most a few SNPs that are significant after adjusting for multiple testing.

Figure 4 shows the QQ plots of the quantiles of the observed $-\log _{10}$ (p-value) versus the theoretical quantiles under the null hypothesis of no association. Departure from the theoretical quantiles implies systematic bias in the data or statistical methods (Laird and Lange, 2011). Specifically bending upward indicates there are too many false positives. Also reported is the genomic control (GC) inflation factor $\lambda$ (Laird and Lange, 2011), which is 
the ratio of the median of the observed test statistics to the median of chi-square distribution with $d_{e}$ degrees of freedom. $\lambda>1$ indicates inflation of type I error. QQ plots and inflation factors in Figure 4 show serious inflation of type I errors under MN and NM models and moderately inflated type I error under DM and NegBin.

Manhattan plots in Figure 5 plot $-\log _{10}(\mathrm{p}$-value) of each SNP under different models (Ziegler and König, 2006). The dashed lines in Manhattan plots indicate the chromosomewide significance level after Bonferroni correction for multiple testing. MN and NM have numerous SNPs that pass the chromosome-wide significance level, indicating inflated type I error. GDM and DM seem to have well controlled type I error and identify some signals on chromosome 8, while NegBin identifies none. Table 8 of Supplementary Materials tabulates the names, positions, minor allele frequency (MAF), and functional annotation of the identified SNPs under the GDM model. Most of the detected SNPs are located in or close to the candidate genes for obesity, cardiovascular diseases, or cancers.

It is hard to draw conclusions based on a sample size of 60 . However, the results seem to conform with our findings in the simulation study in Section 2: (1) choosing a limiting multivariate count model such as multinomial (MN) and negative multinomial (NM) models may inflate type I errors and (2) collapsing counts by simple sums such as in the negative binomial (NegBin) model compromises power for detecting differential expression profiles.

\section{Discussion}

We have investigated GLMs with multivariate categorical responses that, compared to the multinomial-logit model, admit more flexible correlation structures among counts. The RNA-seq simulation example exposes the limitation of the widely used multinomial-logit model. Then we examine three more flexible models for count data: Dirichlet-multinomial, generalized Dirichlet-multinomial, and negative multinomial. Although they do not belong to the exponential family, we show that MLE and regularized estimation can be treated in a unifying framework. The IRPR scheme for MLE is stable, efficient, simple to implement, and enjoys favorable convergence properties. The accelerated proximal gradient algorithm for regularized estimation incorporates various penalties, permitting variable selection, low rank regularization, and entry-wise selection arising from different applications. These regression models provide practitioners more flexible tools for analyzing complex, multivariate count data.

The MLE, testing, and regularized estimation for all four models in Table 2 are implemented in the R package mglm, which is available on CRAN, and a Matlab toolbox, which is available at http://hua-zhou.github.io/softwares/mglm/. We refer readers to the companion paper (Zhang and Zhou, 2015) for more implementation and usage details.

\section{Supplementary Material}

Refer to Web version on PubMed Central for supplementary material. 


\section{Acknowledgments}

The work is partially supported by National Science Foundation (NSF) grant DMS-1310319 and National Institutes of Health (NIH) grants HG006139, GM105785, and GM53275.

\section{References}

Anders S, Huber W. Differential expression analysis for sequence count data. Genome Biology. 2010; 11(10):R106. [PubMed: 20979621]

Baker SG. The multinomial-poisson transformation. Journal of the Royal Statistical Society. Series D (The Statistician). 1994; 43(4):495-504.

Beck A, Teboulle M. A fast iterative shrinkage-thresholding algorithm for linear inverse problems. SIAM J Imaging Sci. 2009; 2(1):183-202.

Chen J, Li H. Variable selection for sparse Dirichlet-multinomial regression with an application to microbiome data analysis. Ann Appl Stat. 2013; 7(1):418-442.

Connor RJ, Mosimann JE. Concepts of independence for proportions with a generalization of the Dirichlet distribution. J Amer Statist Assoc. 1969; 64:194-206.

Dempster AP, Laird NM, Rubin DB. Maximum likelihood from incomplete data via the EM algorithm. J Roy Statist Soc Ser B. 1977; 39(1):1-38. With discussion.

Fan J, Li R. Variable selection via nonconcave penalized likelihood and its oracle properties. J Amer Statist Assoc. 2001; 96(456):1348-1360.

Frank IE, Friedman JH. A statistical view of some chemometrics regression tools. Technometrics. 1993; 35(2):109-135.

Friedman JH, Hastie T, Tibshirani R. Regularization paths for generalized linear models via coordinate descent. Journal of Statistical Software. 2010; 33(1):1-22. [PubMed: 20808728]

Laird, NM., Lange, C. Statistics for Biology and Health. Springer; New York: 2011. The Fundamentals of Modern Statistical Genetics.

Lange K, Hunter DR, Yang I. Optimization transfer using surrogate objective functions. J Comput Graph Statist. 2000; 9(1):1-59. With discussion, and a rejoinder by Hunter and Lange.

Lehmann, EL., Romano, JP. Springer Texts in Statistics. 3. Springer; New York: 2005. Testing Statistical Hypotheses.

McCullagh, P., Nelder, JA. Monographs on Statistics and Applied Probability. Chapman \& Hall; London: 1983. Generalized Linear Models.

Meier L, van de Geer S, Bühlmann P. The group Lasso for logistic regression. J R Stat Soc Ser B Stat Methodol. 2008; 70(1):53-71.

Montgomery S, Sammeth M, Gutierrez-Arcelus M, Lach R, Ingle C, Nisbett J, Guigo R, Dermitzakis E. Transcriptome genetics using second generation sequencing in a caucasian population. Nature. 2010; 464(7289):773-777. [PubMed: 20220756]

Mosimann JE. On the compound multinomial distribution, the multivariate $\beta$-distribution, and correlations among proportions. Biometrika. 1962; 49:65-82.

Nocedal, J., Wright, SJ. Springer Series in Operations Research and Financial Engineering. 2. Springer; New York: 2006. Numerical Optimization.

Robinson MD, McCarthy DJ, Smyth GK. edgeR: a Bioconductor package for differential expression analysis of digital gene expression data. Bioinformatics. 2010; 26(1):139-140. [PubMed: 19910308]

Sossey-Alaoui K, Kitamura E, Head K, Cowell JK. Characterization of FAM10A4, a member of the ST13 tumor suppressor gene family that maps to the $13 \mathrm{q} 14.3$ region associated with b-cell leukemia, multiple myeloma, and prostate cancer. Genomics. 2002; 80(1):5-7. [PubMed: 12079276]

Sun W, Liu Y, Crowley JJ, Chen T-H, Zhou H, Chu H, Huang S, Kuan P-F, Li Y, Miller D, Shaw G, Wu Y, Zhabotynsky V, McMillan L, Zou F, Sullivan PF, Pardo-Manuel de Villena F. IsoDOT detects differential RNA-isoform usage with respect to a categorical or continuous covariate with high sensitivity and specificity. Journal of the American Statistical Association. 2015 accepted. 
Tibshirani R. Regression shrinkage and selection via the lasso. J Roy Statist Soc Ser B. 1996; 58(1): 267-288.

Wang Z, Gerstein M, Snyder M. RNA-Seq: a revolutionary tool for transcriptomics. Nature Reviews Genetics. 2009; 10(1):57-63.

Xu Z, Zhang H, Wang Y, Chang X, Liang Y. $1_{1,2}$ regularization. Science China Information Sciences. 2010; 53(6):1159-1169.

Yuan M, Ekici A, Lu Z, Monteiro R. Dimension reduction and coefficient estimation in multivariate linear regression. J R Stat Soc Ser B Stat Methodol. 2007; 69(3):329-346.

Yuan M, Lin Y. Model selection and estimation in regression with grouped variables. J R Stat Soc Ser B Stat Methodol. 2006; 68(1):49-67.

Zhang CH. Nearly unbiased variable selection under minimax concave penalty. Ann Statist. 2010; 38(2):894-942.

Zhang Y, Zhou H. MGLM: R package and Matlab toolbox for multivariate categorical data analysis. 2015 under revision.

Zhao P, Rocha G, Yu B. The composite absolute penalties family for grouped and hierarchical variable selection. Ann Statist. 2009; 37(6A):3468-3497.

Zhou H, Lange K. MM algorithms for some discrete multivariate distributions. Journal of Computational and Graphical Statistics. 2010; 19:645-665. [PubMed: 20877446]

Zhou H, Lange K, Suchard M. Graphical processing units and high-dimensional optimization. Statistical Science. 2010; 25:311-324. [PubMed: 21847315]

Zhou H, Zhang Y. EM vs MM: a case study. Computational Statistics \& Data Analysis. 2012; 56:3909-3920. [PubMed: 23997380]

Ziegler, A., König, IR. A Statistical Approach to Genetic Epidemiology. Wiley-VCH Verlag GmbH \& Co. KGaA; Weinheim: 2006. Concepts and applications, With a foreword by Robert C. Elston

Zou H, Hastie T. Regularization and variable selection via the elastic net. J R Stat Soc Ser B Stat Methodol. 2005; 67(2):301-320. 

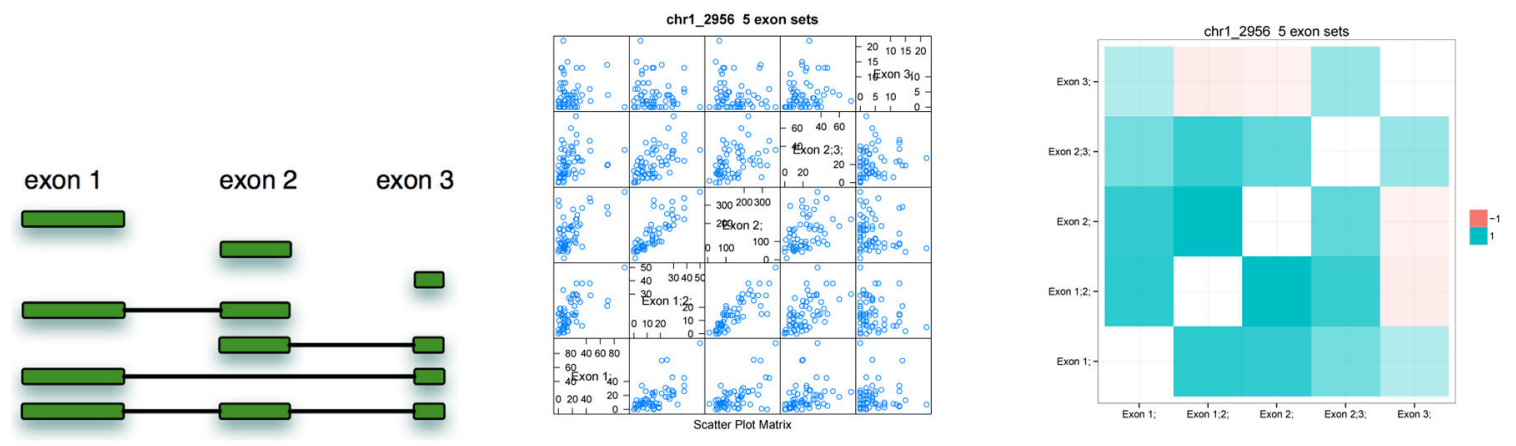

Figure 1.

Left: A gene with 3 exons and 7 possible isoforms. Middle and right: Pairwise scatter plots and correlations of exon counts of a gene with 5 exon sets. 


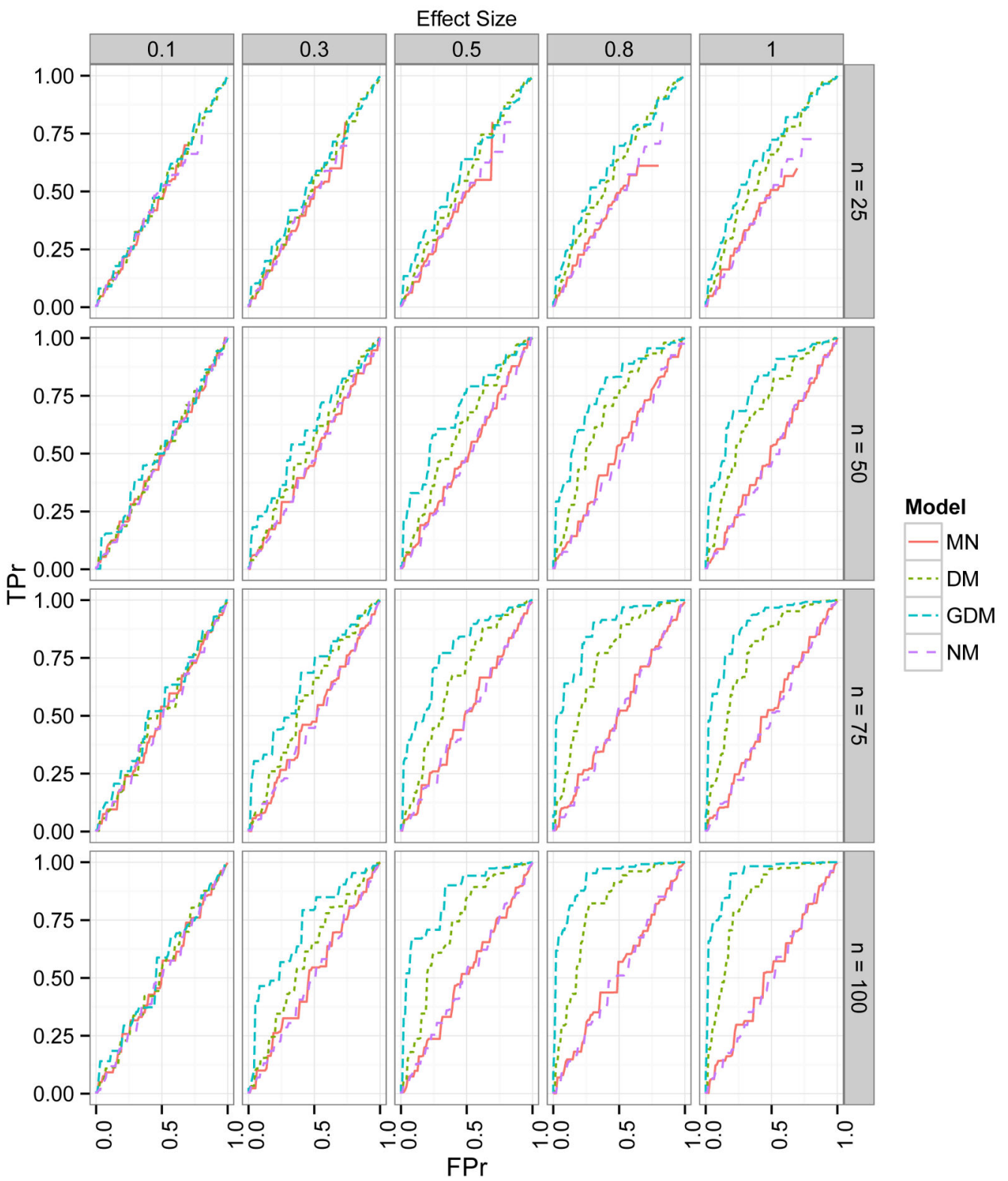

Figure 2.

ROC curves from the group penalized estimation by the multinomial (MN), Dirichletmultinomial (DM), and generalized Dirichlet-multinomial (GDM) regression models. ROC curves are summarized from 300 simulation replicates. Responses are generated from the generalized Dirichlet-multinomial (GDM) model. 


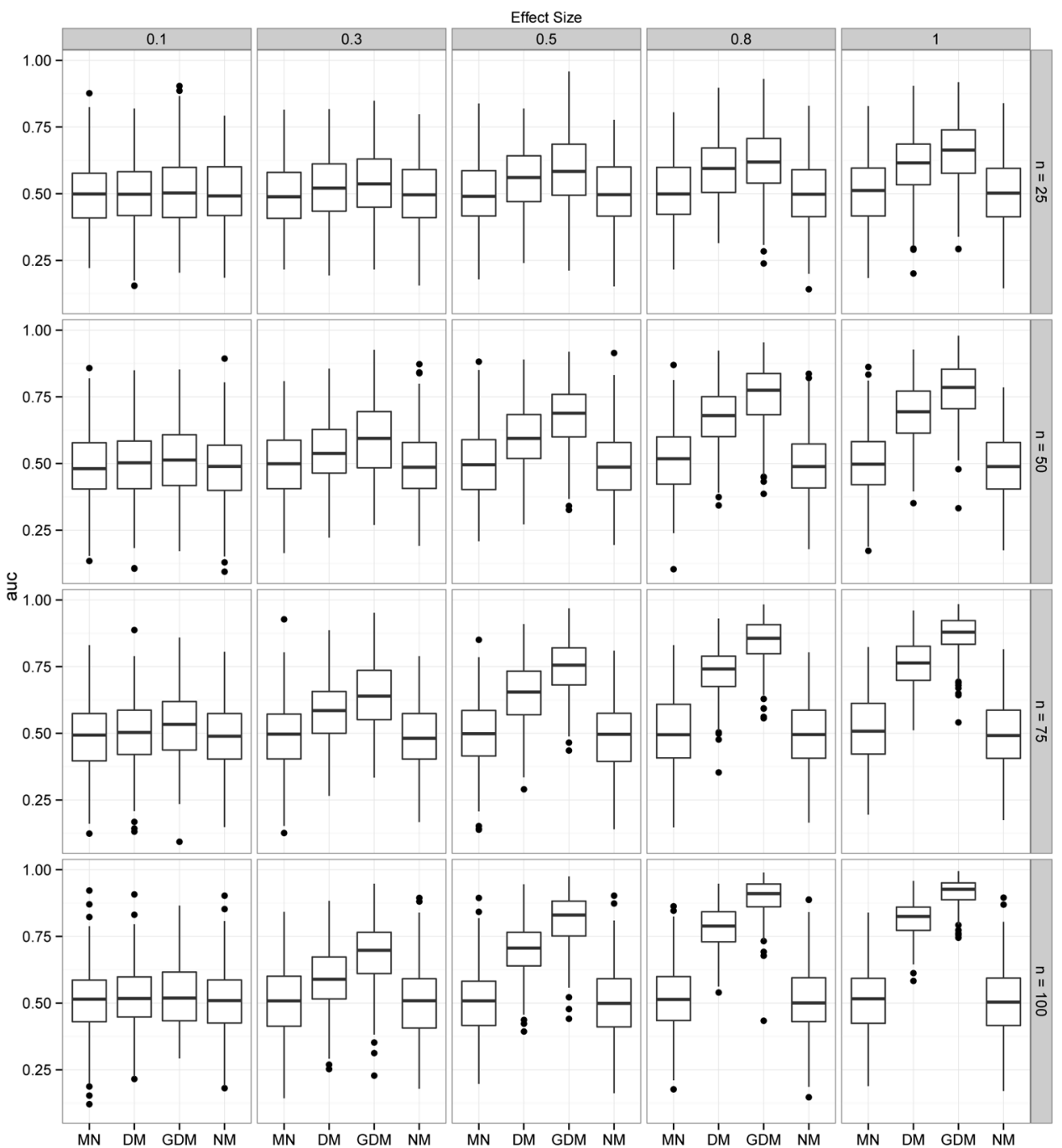

Figure 3.

Box plots of AUCs from the group penalized estimation by the multinomial (MN), Dirichletmultinomial (DM), generalized Dirichlet-multinomial (GDM) and negative multinomial (NM) regression models, based on 300 simulation replicates. Responses are generated from the GDM model. 


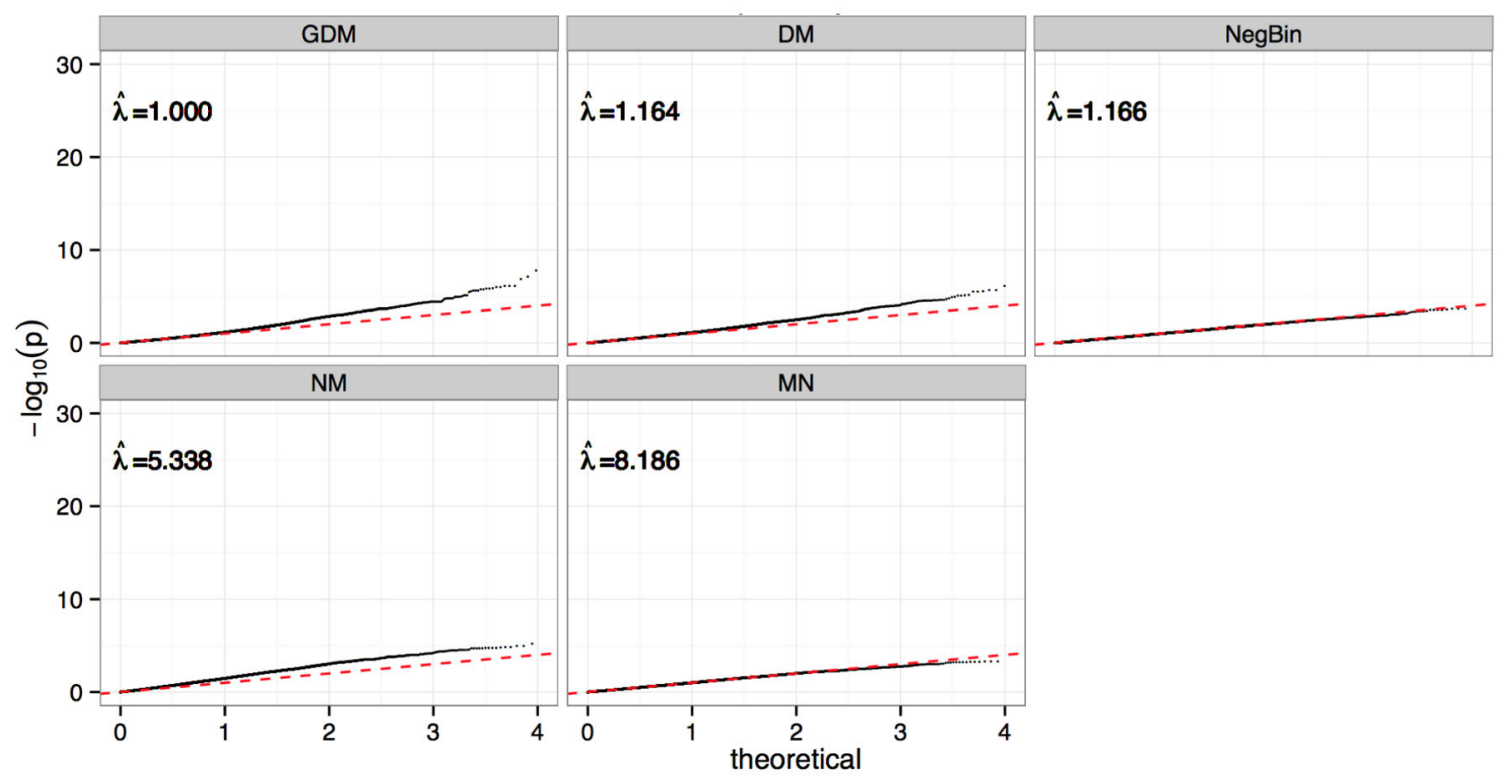

Figure 4.

QQ plots of eQTL analysis of ST13P6 using MN, DM, GDM and NM regressions. $\hat{\lambda}$ are the estimated genomic control (GC) inflation factor. 


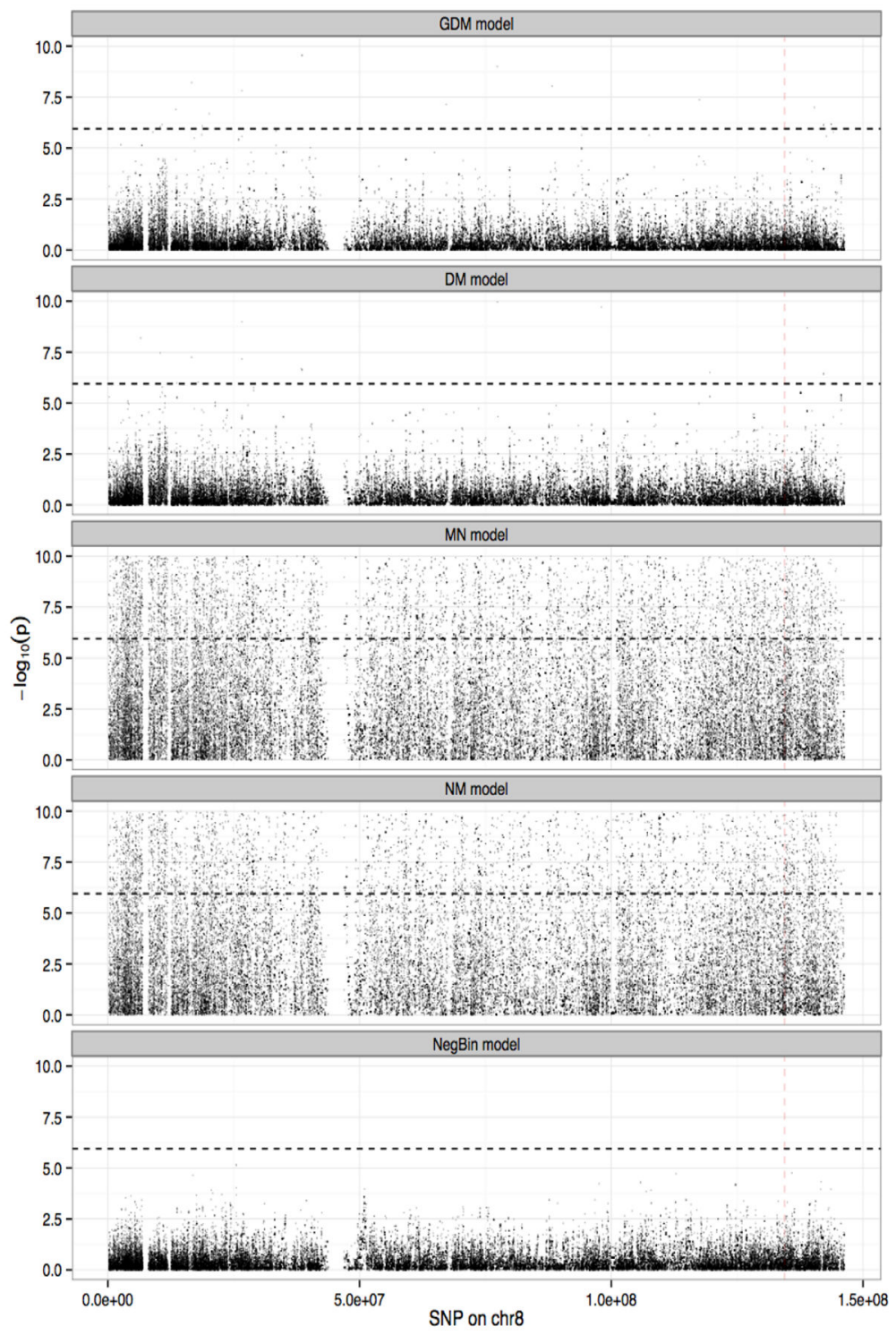

Figure 5.

Manhattan plots of GDM, DM, MN, NM and NegBin regressions for eQTL analysis of ST13P6. Dashed lines are chromosome-8-wide significance level. 


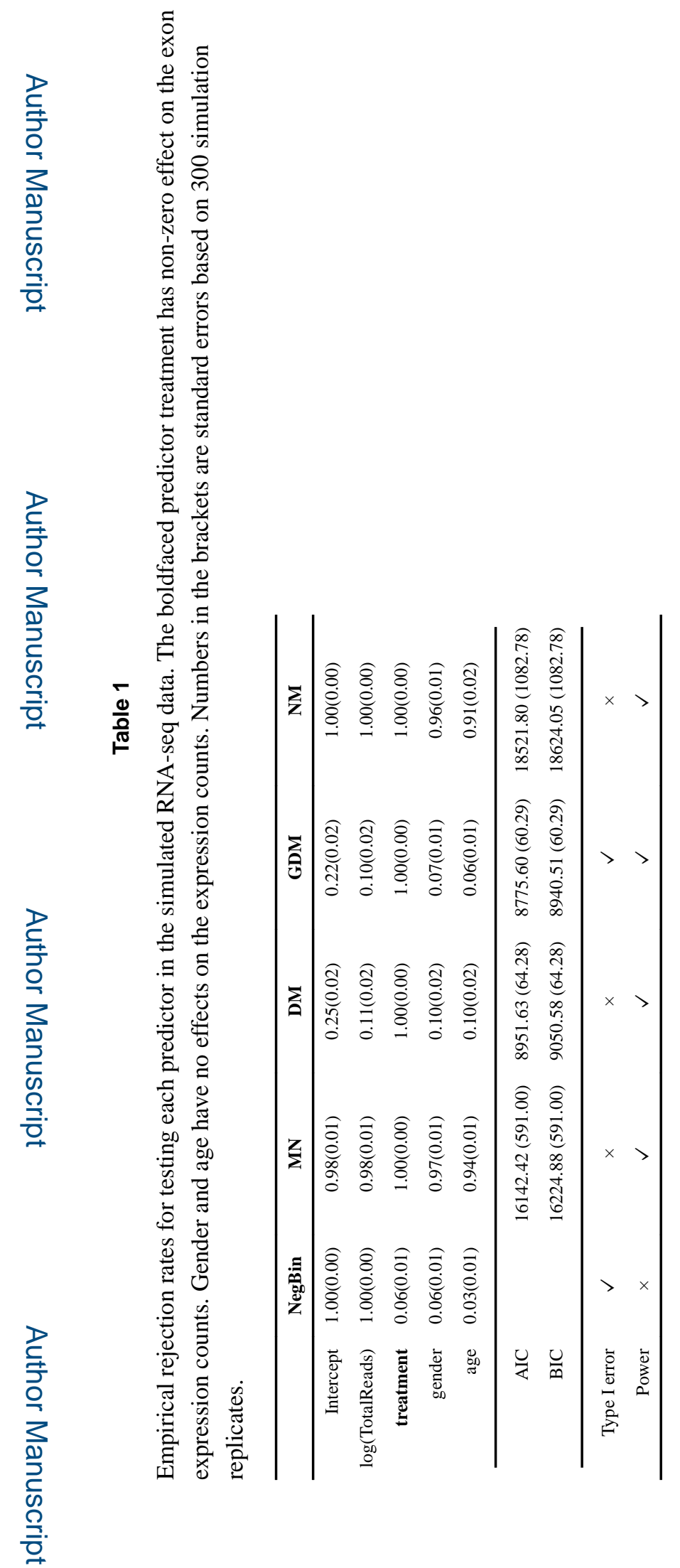

J Comput Graph Stat. Author manuscript; available in PMC 2018 February 16. 


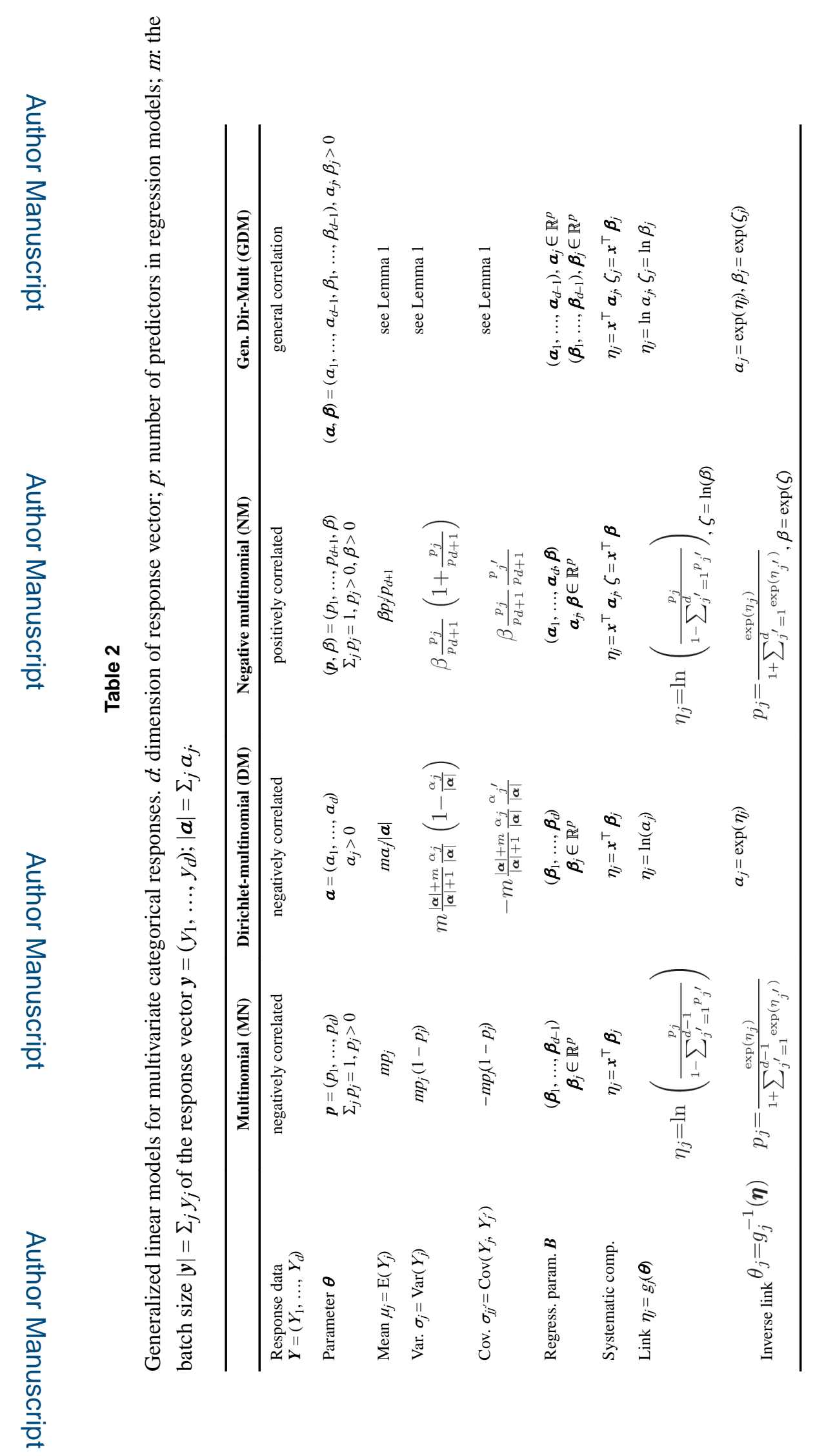

J Comput Graph Stat. Author manuscript; available in PMC 2018 February 16. 


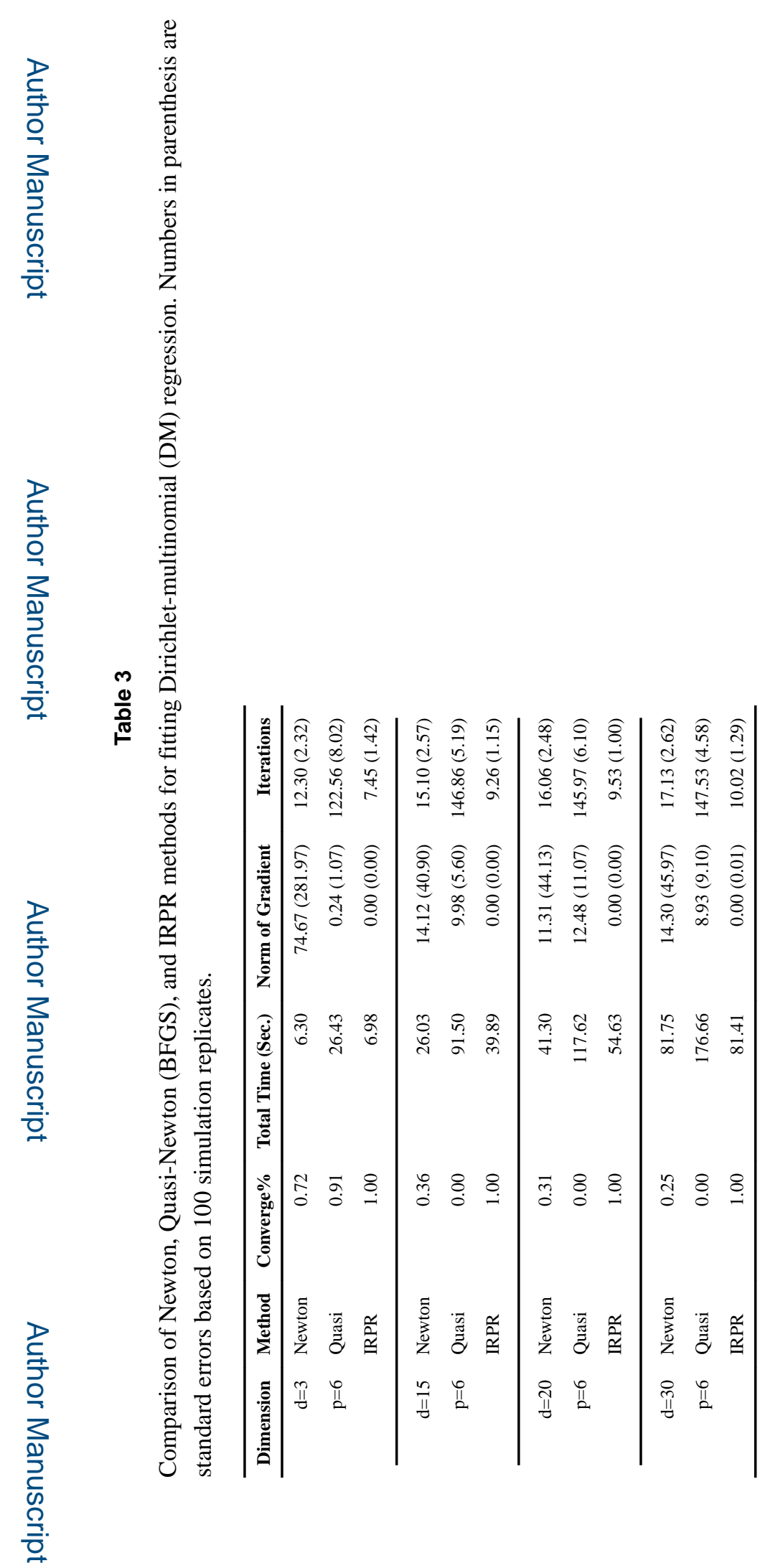




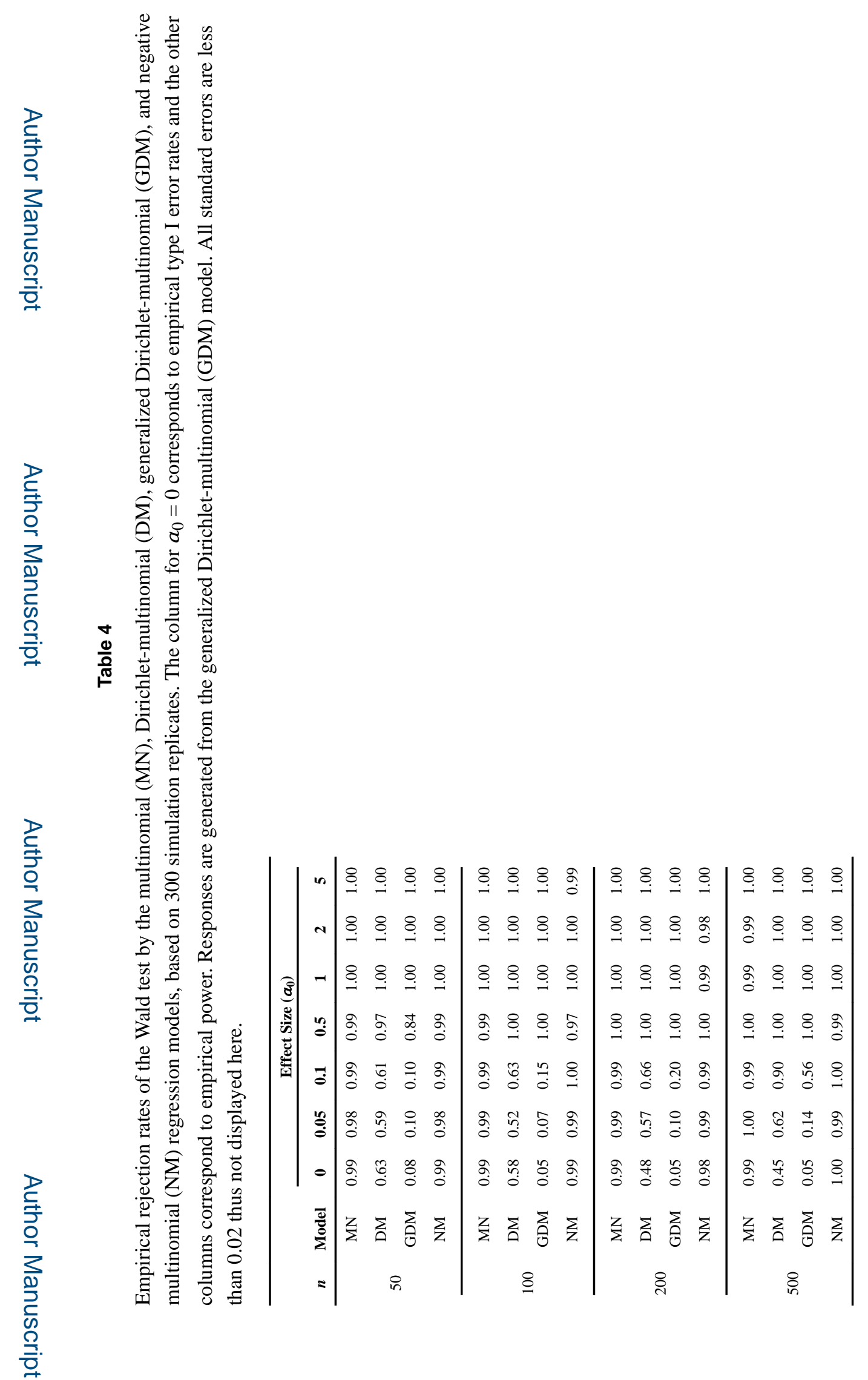

J Comput Graph Stat. Author manuscript; available in PMC 2018 February 16. 